\title{
Milder Preparation of a $\gamma$-Azido- $\alpha$-diazo- $\beta$-keto Ester by Consecutive Introduction of Azido and Diazo Groups
}

\section{Luiz Gustavo Dutra (PG), ${ }^{*}$ Marcus Mandolesi Sá (PQ)}

Departamento de Química, Universidade Federal de Santa Catarina, Florianópolis/SC, 88040-900, Brasil

${ }^{*}$ Corresponding author. Tel. +55-48-37219967; Fax: +55-48-37216850

*e-mail: dutralg@yahoo.com.br

Keywords: Azido esters, Diazo carbonyl, Diazo transfer

\section{INTRODUCTION}

Diazo compounds have been used extensively as precursors of metal carbene intermediates, which can subsequently undergo diverse transformations such as $\mathrm{X}-\mathrm{H}$ insertion $(\mathrm{X}=\mathrm{C}, \mathrm{O}, \mathrm{S}, \mathrm{N})$, and cyclopropanation. ${ }^{1}$ Organic azides are valuable intermediates for the synthesis of many nitrogencontaining molecules, ${ }^{2}$ including heterocycles and natural products. ${ }^{3}$ Although the isoelectronic azido and diazo functionalities are synthetically useful, the chemical behavior of compounds decorated with both azido and diazo groups remains unexplored. ${ }^{4}$

Some years ago, we reported ${ }^{5}$ the synthesis of $\gamma$ azido- $\alpha$-diazo- $\beta$-keto esters and the chemoselective transformation of their multiple functional groups that ultimately gave more elaborated structures. ${ }^{6}$ The key azido diazo building block 1 was originally prepared in 2 steps by coupling the diazomercurial 2 with bromoacetyl bromide under anhydrous conditions followed by treating the bromo diazo intermediate $\mathbf{3}$ with azide anion ${ }^{5}$ (Scheme 1, Red route). Although this methodology furnished the expected product 1 in high yields and mild conditions, the diazomercurial 2 is not commercial and harmful to the health and environment. Herein, we report our initial achievements through a more simple and safe methodology for the synthesis of 1 starting from the commercially available ethyl 4-chloroacetoacetate (4) (Scheme 1, Blue route).

\section{RESULTS AND DISCUSSION}

The $\gamma$-chloro- $\alpha$-diazo- $\beta$-keto ester 5 was prepared from 4 by a diazo transfer reaction using tosyl azide $\left(\mathrm{TsN}_{3}\right)$ in basic medium. A variety of conditions was studied, including different combinations of base (triethylamine [TEA], $\mathrm{N}$-methylmorpholine, $\mathrm{K}_{2} \mathrm{CO}_{3}$ ) and solvent (THF, acetone, acetonitrile). While the use of TEA in THF led to a clean formation of $\mathbf{5}$, the recovery mass was consistently low, possibly due to an extensive loss of product during the aqueous work-up. More promising results were achieved with the bulkier base $\mathrm{N}, \mathrm{N}$-diisopropylethylamine (DIPEA) in THF for $24 \mathrm{~h}$ followed by quenching the reaction with $\mathrm{HCl}$ before the basic aqueous work-up, giving the chloro diazo ester 5 in $50-70 \%$ yield.
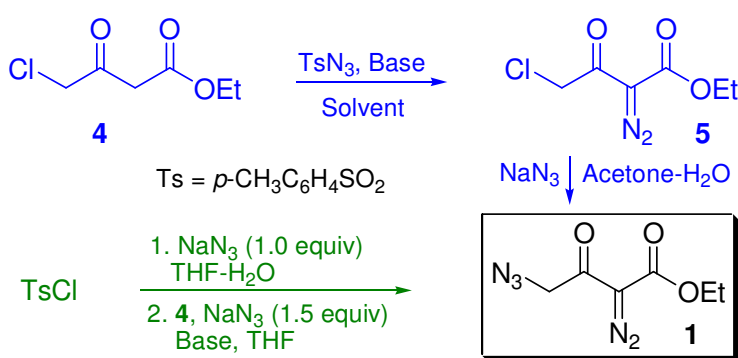

$\mathrm{NaN}_{3} \downarrow$ Acetone- $\mathrm{H}_{2} \mathrm{O}$

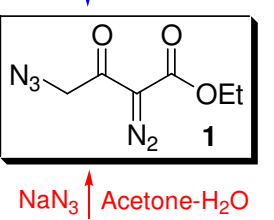

$2\left(\prod_{N_{2}}^{O}\right)_{2}$

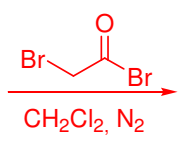

$\prod_{\prod_{2}}^{O} \underbrace{O}_{3}$

Scheme 1. Preparation of azido diazo ester 1.

The subsequent preparation of azido diazo ester 1 from $\mathbf{5}$ by chlorine displacement with azide in aqueous acetone for $24 \mathrm{~h}$ gave the expected product in high yield. Diazo compounds $\mathbf{1}$ and $\mathbf{5}$ were characterized by $\mathrm{IR}$ and ${ }^{1} \mathrm{H}$ NMR, and their spectroscopic data were in agreement with those already published. ${ }^{5}$

Finally, preliminary results indicated that azido diazo ester 1 can be prepared in one pot from $\mathbf{4}$ and tosyl chloride, by first generating $\mathrm{TsN}_{3}$ in situ followed by consecutive diazo transfer and displacement with azide (Scheme 1, Green route).

\section{CONCLUSION}

Preparation of azido diazo ester 1 from 4 by a diazo transfer reaction and subsequent nucleophilic displacement is a promising methodology. This simple protocol can be adapted to a one-pot process without isolation of any intermediates. Further studies are in progress to optimize the reaction conditions.

\section{ACKNOWLEDGEMENTS}

UFSC, CAPES, CNPq, FAPESC

\section{REFERENCES}

${ }^{1}$ Zhang, Z.; Wang, J. Tetrahedron 2008, 64, 6577

Bräse, S.; Gil, C.; Knepper, K.; Zimmermann, V. Angew. Chem. Int. Ed. 2005, 44, 5188.

${ }^{3}$ Mazal, C.; Jonas, J.; Zak, Z. Tetrahedron 2002, 58, 2729.

${ }^{4}$ Sá, M. M.; Silveira, G. P.; Bortoluzzi, A. J.; Padwa, A. Tetrahedron 2003, 59, 5441 and references cited herein.

${ }^{5}$ Padwa, A.; Sá, M. M.; Weingarten, M. D. Tetrahedron 1997, 53, 2371.

${ }^{6}$ Padwa, A.; Sá, M. M. J. Braz. Chem. Soc. 1999, 10, 231.

$14^{\text {th }}$ Brazilian Meeting on Organic Synthesis $-14^{\text {th }}$ BMOS - September 01-05, 2011-Brasilia, Brazil 